\title{
No political will to seek innovative contraception
}

\section{Focus instead on other reproductive issues, which may make birth-control superfluous.}

Sir - Your Outlook supplement on fertility includes a Commentary article by J. F. Strauss and M. Kafrissen, "Waiting for the second coming" (Nature 432, 43-45; 2004), which is either an extraordinarily naive recommendation for developing fundamentally new "safer, more effective and more userfriendly" contraceptives or a disingenuous plea for more money. In this field, pouring additional funds into research without addressing the underlying problems is as illogical as getting nine women pregnant and expecting a baby in one month.

The opening sentence, "Even developed countries have a staggeringly high incidence of unplanned pregnancies", leads the authors to conclude that currently available contraceptives "are simply not meeting the needs of society". Yet it is primarily through the use of the Pill, intrauterine device (IUD), condom, sterilization and abortion that the reproduction rate in every European country except Albania and Malta is now below replacement level. Even in Japan, where the Pill was legalized only in 1999, the rate fell below replacement level years ago. A combination of almost static contraceptive 'hardware' and the more dynamically changing 'software' - the cultural, economic, public health, educational, political (notably in
China) and women's-rights issues — has proved crucial to the decline in much of the world's population growth.

Hence it is shameless grantsmanship to claim that promising leads will result from more money for "forays into 'glycomics' and 'lipidomics' [and] new technologies for large-scale analysis of carbohydrates and lipids". Such leads will have nothing to do with practical contraception within the reproductive lifetime of any living infant.

Of the 20 largest pharmaceutical companies in the world, only two (Johnson \& Johnson and Wyeth) market female contraceptives and do a modest amount of $R \& D$ to improve existing steroid contraceptives. None of the 20 is active in the male-contraceptive field. None has been willing to continue contraceptivevaccine research initiated by the World Health Organization. The types of incentives listed by the Commentary authors to encourage a re-entry of the pharmaceutical industry, now fixated on blockbuster drugs for geriatric populations, are largely a rehash of earlier proposals (see C. Djerassi Science 245, 356-361; 1989) for which there is no political will for implementation.

There are no short cuts to establishing the authors" illusory goal of "safer" birth control (safer than condoms, IUDs or sterilization?) without demonstrating clinically that consumption of a fundamentally new contraceptive for $10-20$ years by women or 20-40 years by men is indeed safe. Who will pay the hundreds of millions of dollars required for such studies?

It makes more sense to focus on the reproductive realities described by other articles in your Fertility Outlook: research on conception, infection and the extension of female fertility. Or the enormous practical advances made in assisted reproduction, which do not require the pharmaceutical industry for research or practical implementation. Consider that in China and the United States, sterilization has now surpassed the Pill and other contraceptives among married couples.

As sex and fertilization become increasingly separated, the cryopreservation of semen by young men followed by sterilization and subsequent use of artificial insemination to create their future one or two children (see C. Djerassi and S. P. Leibo Nature 370, 11-12; 1994) may one day make contraception superfluous. Carl Djerassi

Department of Chemistry, Stanford University, Stanford, California 94305-5080, USA

\section{Emissions control needs atmospheric verification}

Sir - As your News Feature "The carbon game" (Nature 432, 268-270; 2004) makes clear, the start of the European Emissions Trading Scheme may be a route to controlling greenhouse gases. Coupled with the United Nations' Clean Development Mechanism to encourage participation by the poorer nations, it may indeed work.

However, independent atmospheric verification of emissions has long been neglected. The system relies on producers reporting their own greenhouse emissions. Until now, there has been no financial penalty for producing emissions and no benefit from carbon sequestration. Now that money enters the picture, so also can fraud.

Today's emitters of greenhouse gases report their emissions; the information is then passed on to national and international databases. Under the new scheme, there will now be an incentive to under-report emissions at every stage. Equally, those sequestering carbon will be tempted to exaggerate. Despite diligent independent controls, doubts will arise at all levels. Even when there is no intent to misreport, companies, regions and nations will suspect each other.

"Trust but verify" as Ronald Reagan used to say, quoting a Russian motto. The Nuclear Test Ban Treaty created trust by diligent verification. Atmospheric monitoring detected fallout, and a global network of seismographs was set up in order to detect explosions. Trust came, bomb tests ended. Plate tectonics was a surprise bonus.

The Kyoto Protocol lacks this. Verification is not expensive, but atmospheric monitoring at present is inadequate. A modest but effective multinational programme to assess net carbon-gas emissions would not cost much. Satellites give broad-brush imagery, but the main need is for very precise measurements on the ground, which are not expensive to carry out. For methane, modelling based on careful monitoring of concentrations and isotopes is already verifying national emission inventories by source type and seasonality.

Most of the monitoring load falls on the United States. There are a few exceptions in the form of some excellent national programmes, and the European Union has programmes such as CarboEurope. But many non-US programmes have only short-term funding, despite the need for continuity. The British contribution is minimal. There is little on the tropical landmasses.

Other nations must do more to share in detailed long-term understanding of carbon budgets, including uptakes by the terrestrial biosphere. The Commonwealth 'club', led by strong Australian and Canadian programmes, could help in the tropics.

To win the trust of the United States, China and India, whose emissions growth will damage us all, we need to verify emissions and uptakes accurately and in detail.

\section{Euan Nisbet}

Atmospheric Laboratory, Department of Geology, Royal Holloway, Egham, Surrey TW20 OEX, UK

\section{correspondence}

Contributions to Correspondence may be submitted to corres@nature.com. They should be no longer than 500 words, and ideally shorter. Published contributions are edited. 